\title{
Erratum
}

\section{Extrasolar planets and brown dwarfs around A-F type stars}

\section{A planetary system found with HARPS around the F6IV-V star HD 60532}

\author{
M. Desort ${ }^{1}$, A.-M. Lagrange ${ }^{1}$, F. Galland ${ }^{1}$, H. Beust ${ }^{1}$, S. Udry ${ }^{2}$, M. Mayor ${ }^{2}$, and G. Lo Curto ${ }^{3}$ \\ 1 Laboratoire d'Astrophysique de Grenoble, UMR5571 CNRS, Université Joseph Fourier, BP 53, 38041 Grenoble Cedex 9, France \\ e-mail: mdesort@obs.ujf-grenoble.fr \\ 2 Observatoire de Genève, Université de Genève, 51 chemin des Maillettes, 1290 Sauverny, Switzerland \\ 3 European Southern Observatory, Alonso de Cordova 3107, Vitacura, Santiago, Chile
}

A\&A 491, 883-888 (2008), DOI: 10.1051/0004-6361:200810241

\begin{abstract}
The dynamical analysis in the original paper was erroneous due to a mismatch in the choice of angular parameters. The calculations had been made by assuming a pole-on $\left(\sin i=0\right.$ ) instead of an edge-on $\sin i=1$ orbit. In this framework, $\Omega_{c}-\Omega_{b}$ is just the mutual inclination between the orbital planes of the two planets. We also correct some stellar parameters given in the original paper $\left(\log g=+3.83,[\mathrm{Fe} / \mathrm{H}]_{\text {updated }}=-0.26\right)$.
\end{abstract}

Key words. techniques: radial velocities - stars: early-type - stars: planetary systems - stars: oscillations - individual: HD 60532 errata, addenda

In the original paper the calculations had been made by assuming a pole-on $(\sin i=0)$ instead of an edge-on $(\sin i=1)$ orbit. The dynamical study has been made again, but this time by assuming coplanarity of the orbits, hence $\Omega_{c}=\Omega_{b}$. The figures are changed but the main conclusions remain. Over $10^{8} \mathrm{yr}$, the planetary system is chaotic but does not indicate any instability. The semi-major axes of the two planets oscillate between $0.754 \mathrm{AU}$ and $0.752 \mathrm{AU}$ for planet $b$, and between $1.568 \mathrm{AU}$ and $1.595 \mathrm{AU}$ for planet $c$. The eccentricity of planet $b$ oscillates between 0.118 and 0.3 , and that of planet $c$ between 0.015 and 0.141. As before, we show that, given the error bars, the secular evolution of the semi-major axis of planet $b$ should be detectable within $\sim 10$ years from now. This would constitute a strong indication of a resonant configuration. The sense of this variation is not constrained, because of the error bar on the argument of the planet $c$ periastron. Figure 2 shows the secular evolution of the semi-major axis of planet $b\left(a_{b}\right)$ in the same conditions as above, but for an initial choice of $\omega_{c}=-280^{\circ}$ instead of $-209^{\circ}$. The initial evolution sense is reversed compared to Fig. 1.

As in the initial calculations, the size of the error bars in Table 2 does even not ensure that the orbital configuration is actually resonant, but here again in non resonant configurations (Fig. 4), the variations of the semi-major axis of planet $b$ achieve a much lower amplitude than in the resonant case.
Our basic conclusions are thus unchanged: i) the resonant configuration cannot be stated, but it is probable; ii) the system is significantly chaotic; iii) in a resonant configuration, we should be able to detect semi-major axis variations in planet $b$ 's motion within $\sim 10$ yrs.

Recently, a global analysis of this system by Laskar \& Correia (2009) confirmed the resonant status, using numerical integration and frequency analysis. In fact, non resonant systems appear less stable than resonant ones of Gyr timescales. This further indicates a resonant configuration.

We must also correct the $\log g$ of the star, which is +3.83 . And we can update the estimated metallicity, which is now -0.26 according to new calibrations of the data from Holmberg et al. (2007), thus slightly more metallic than before. An estimation from Gray et al. (2006) (from which we took the $\log g$ ) gives $[\mathrm{Fe} / \mathrm{H}]=-0.05$.

Acknowledgements. We thank Daniel Fabrycky, Jacques Laskar, and Alexandre Correia for pointing out the dynamical inconsistencies of the initial version of the paper, and the referee for the stellar parameter corrections.

\section{References}

Holmberg J., Nordström B., \& Andersen J., 2007, A\&A, 475, 519

Laskar J., \& Correia A.C.M. 2009, A\&A, 496, L5 

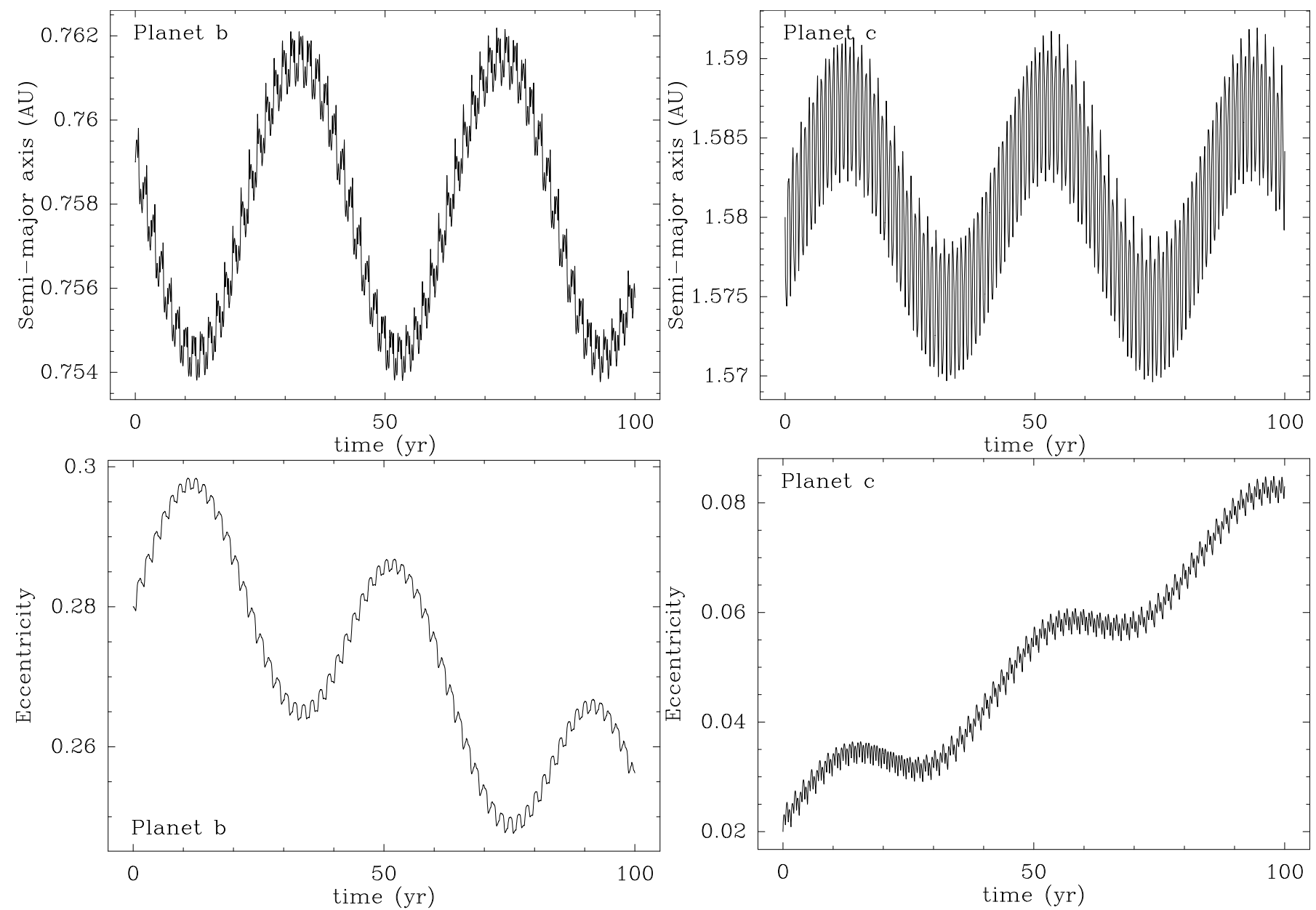

Fig. 1. Orbital evolution over $100 \mathrm{yr}$ of the semi-major axes (top) and eccentricities (bottom) for planets $b$ (left) and $c$ (right), under their mutual perturbations, in a 3:1 resonance configuration.

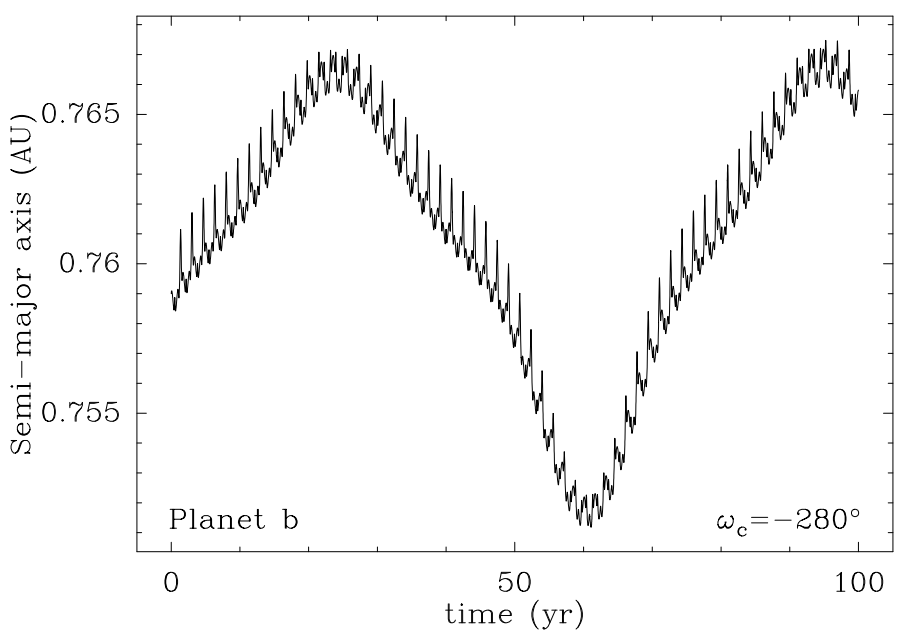

Fig. 2. Evolution of semi-major axis if planet $b$ in the same conditions as in Fig. 1, but assuming an initial $\omega_{c}=-280^{\circ}$ instead of $-209^{\circ}$.

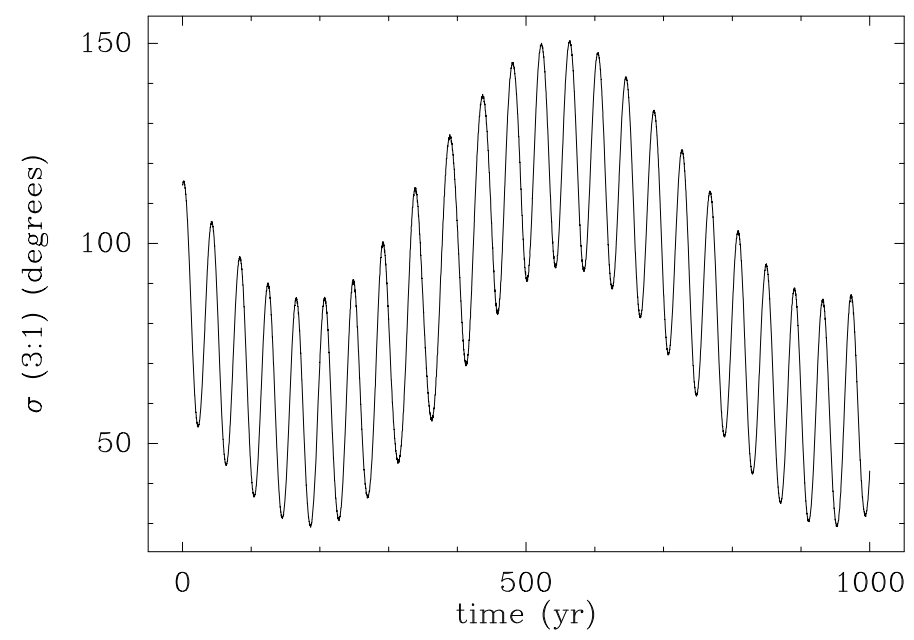

Fig. 3. Evolution of the 3:1 critical argument $\sigma$ over $1000 \mathrm{yr}$, in the same condition as described in Fig. 1. We note the $\sigma$-libration characteristic for resonant motion. 

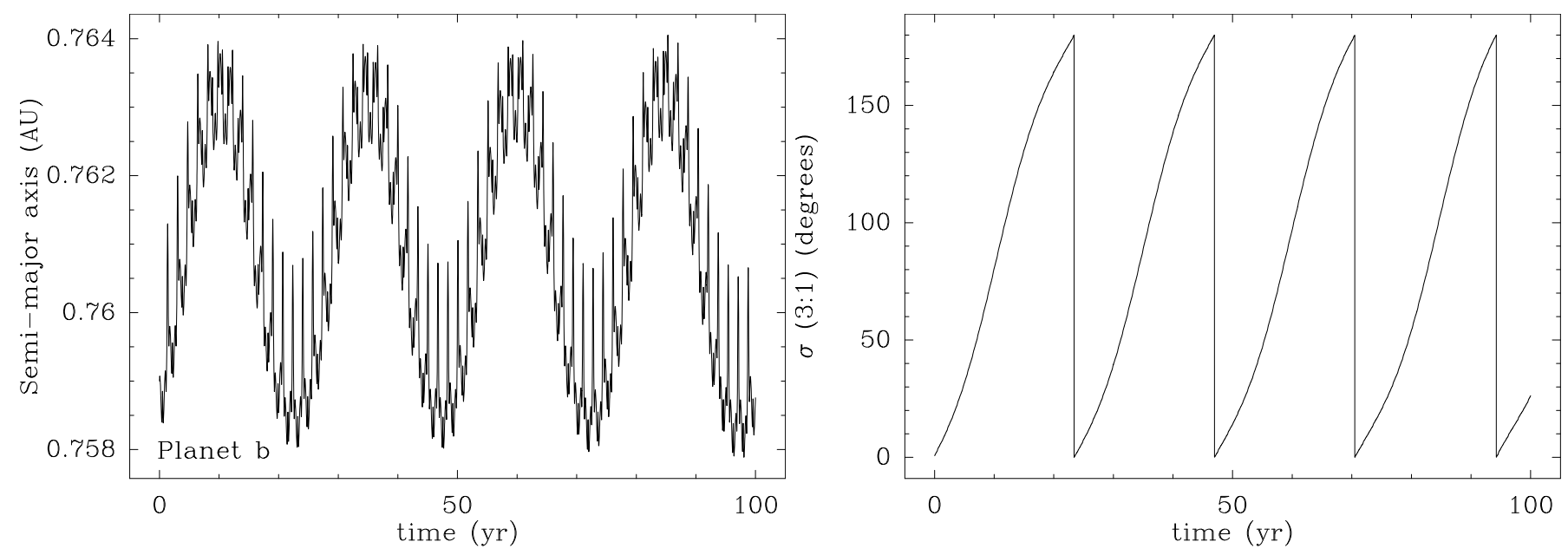

Fig. 4. Evolution of the semi-major axes of planet $b$ and of the critical angle $\sigma$ for 3:1 resonance in the same conditions as described in Fig. 1, but with $a_{c}=1.56 \mathrm{AU}$. This is a non-resonant configuration. 